\title{
TESTING OF HOM COUPLER DESIGNS ON A SINGLE CELL NIOBIUM CAVITY*
}

\author{
P. Kneisel ${ }^{\#}$, G. Ciovati, G.R. Myneni and G. Wu, TJNAF, Newport News, VA, USA \\ J. Sekutowicz, DESY, 22603 Hamburg, Germany
}

\begin{abstract}
Coaxial higher order mode (HOM) couplers were developed initially for HERA cavities and subsequently for TESLA cavities. They were adopted later for SNS and Jlab upgrade cavities. The principle of operation is the rejection of the fundamental mode by the tunable filter and the transmission of the HOMs. It has been recognized recently that for continuous wave or high duty factor applications of the TESLA coupler the output pick-up probe must stay superconducting in order to avoid its heating by the fundamental mode residual magnetic field leading to deterioration of the cavity quality factor. In addition, the thermal conduction of existing $\mathrm{rf}$ feedthrough designs is only marginally sufficient to keep even the niobium probe tip superconducting in $\mathrm{cw}$ operation. We have equipped a single-cell niobium cavity with the modified HOM couplers and tested the new designs by measuring $\mathrm{Q}$ vs $\mathrm{E}_{\text {acc }}$ behavior at $2 \mathrm{~K}$ for different feedthroughs and probe tip materials.
\end{abstract}

\section{INTRODUCTION}

The coaxial HOM coupler was originally developed for the $500 \mathrm{MHz}$ HERA cavities in 1985. This coupler provided damping of dangerous modes to $\mathrm{Q}_{\mathrm{ext}}$ values below 1000 .

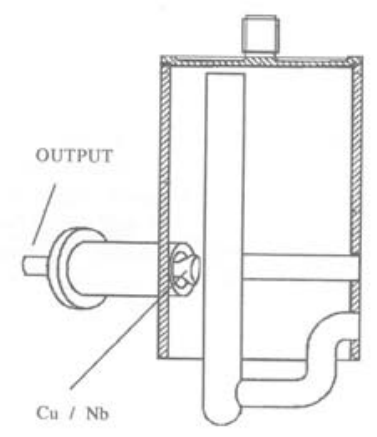

Figure 1: HOM coupler schematic (from Ref. [1]).

Later, this coupler design as schematically shown in Figure 1 was adopted for the TESLA cavities after modifications to the frequency of $1300 \mathrm{MHz}$ [1]. Because of its successful use in these two projects, it was later adopted for the SNS project and the CEBAF upgrade cavities. The principal of operation is as follows. The inductance of the coupling loop and the capacitance between endplate of the can together with coaxial line

\footnotetext{
* Supported in part by DOE contract DE-AC05-84ER40150

"kneisel@jlab.org
}

between endplate and the output form a tunable rejection filter. The notch of the filter can be tuned such that the fundamental mode frequency is rejected with $\sim-100 \mathrm{~dB}$, while the HOM frequencies can pass and are coupled out by the coupling probe to an external load. There is however quite some stored energy in the coupler with the maximum magnetic field near the pick-up probe tip, while the electric field in this area is minimized. The induced currents in the probe tip associated with the magnetic field can lead to heating of the probe tip, if the surface resistance is high and if the generated heat cannot be transferred efficiently to the surrounding environment, e.g.. heat sinking at cryogenic temperatures.

In 2003 during the prototype development of cavities for the CEBAF's upgrade we discovered that already at a very low accelerating gradient of $\sim 3 \mathrm{MV} / \mathrm{m}$ the cavity $\mathrm{Q}$ begun to drop rapidly with increasing field in the cavity and it took up to $1 \mathrm{hr}$ after the rf was switched off for the cavity $Q$ to recover as shown in Figure 2. This was a clear indication that the feedthrough/probe tip configuration used in these tests (probe tip was made of copper) did not provide sufficient heat transfer and that this additional losses were loading the cavity $\mathrm{Q}$. The long recovery time was another indication of very poor thermal properties of the feedthrough. The feedthrough flange was in direct contact with the He bath at $2 \mathrm{~K}$ and the probe tip was screwed on the feedthrough end.

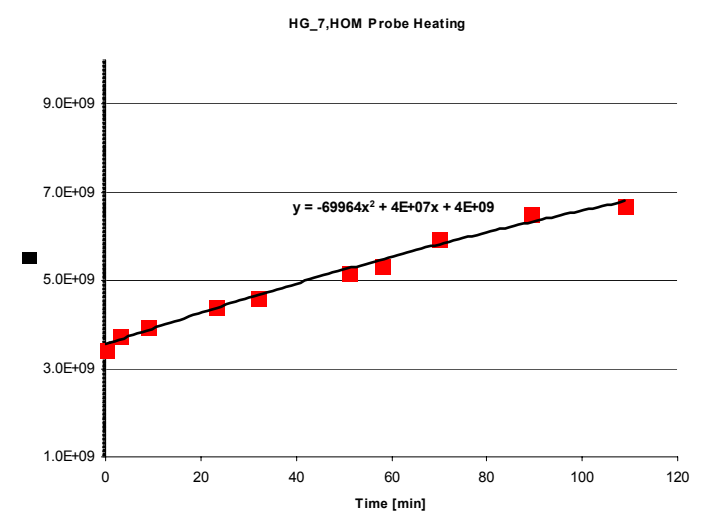

Figure2: Recovery of the Q value of a 7-cell Upgrade cavity after heating of the probe.

\section{THERMAL CALCULATIONS AND MEASUREMENTS ON FEEDTHROUGHS}

Following this discovery, an attempt was made to estimate the RF heating of the HOM coupler output pin in 
the upgrade cavities. The ratio of the magnetic fields were measured at the positions indicated in Figure 3

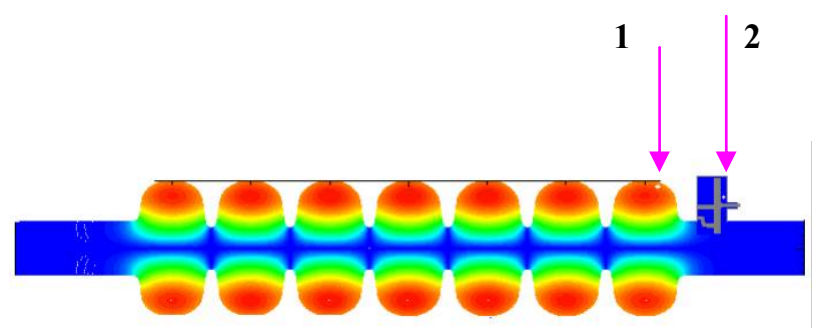

Figure3: Magnetic field measurements on 7-cell cavity.

This ratio is 0.035 and at the nominal field of $\mathrm{E}_{\mathrm{acc}} \sim 20$ $\mathrm{MV} / \mathrm{m}$ a power of 7 Watts is dissipated in the copper pin. It became clear that the coupler pin needs to be superconducting, that an improved feedthrough with better heat flow was needed and that the heat influx from the output $\mathrm{rf}$ cable to the feedthrough had to be minimized. For comparison, in the TTF cavities the pin is made of niobium and the cavities are operated with a $1 \%$ duty cycle; the heating by rf is app. a factor of $10^{6}$ smaller than for the upgrade cavities.

Thermal measurements on the original rf feedthrough were carried out in a set-up shown in Figure 4: the feedthrough is clamped into a copper block connected through the endplate of a vacuum can with the helium bath. A heater is attached to the probe tip and 2 thermometers on the probe tip and the feedthrough flange can measure the $\Delta \mathrm{T}$ along the tip.

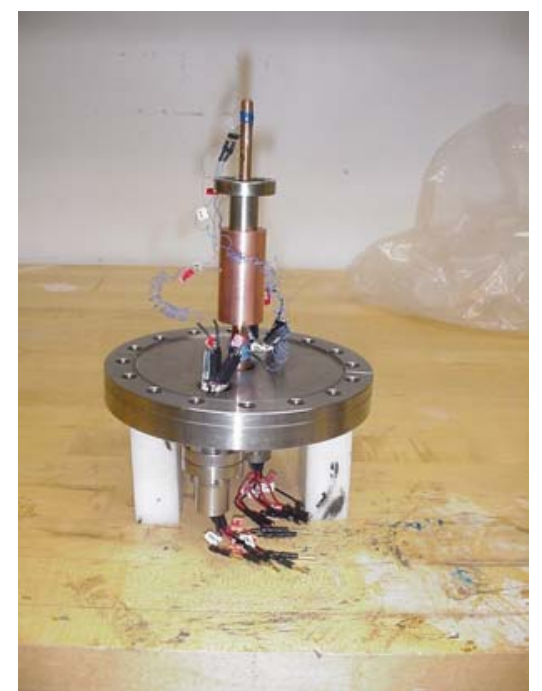

Figure 4: Thermal measurement set-up for measuring the heat transfer through the feedthrough into the He bath.

The measurements, backed up by thermal model calculations [2] showed that already at a dissipated power of $1-2 \mathrm{~mW}$ the tip temperature of the copper tip reached $\mathrm{T}>10 \mathrm{~K}$; with a Nb tip soldered to a feedthrough used for the TESLA cavities [Kyocera] $\geq 10 \mathrm{~mW}$ could be tolerated.

It became clear that for the $\mathrm{cw}$ operation of the upgrade cavities at a gradient of $\sim 20 \mathrm{MV} / \mathrm{m}$ either the feedthrough thermal performance had to be improved or the HOM coupler configuration had to be modified.

Both options were pursued: a thermally more stable feedthrough with a sapphire window was developed "in house" [3] and the tip geometry was optimized using HFSS as shown in Figure 5 [4]; secondly, the loop part of the HOM coupler was modified as shown in Figure 6. The extension of the coupling loop towards the probe tip changes the field distribution at the probe tip and less power is dissipated.
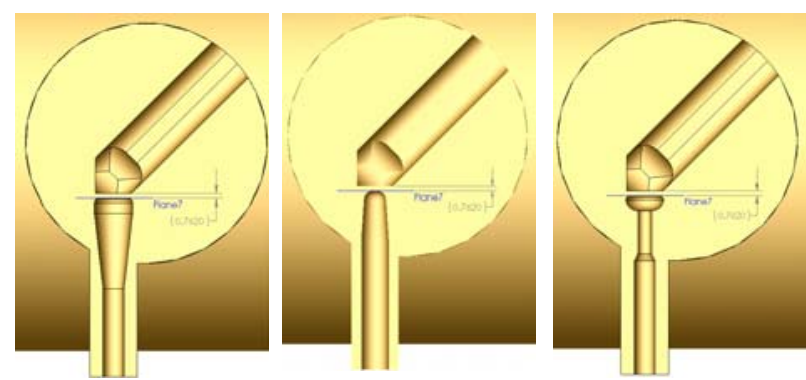

Figure 5: Different Probe tip geometries; the lowest losses were obtained with the tapered tip in the center of the figure.

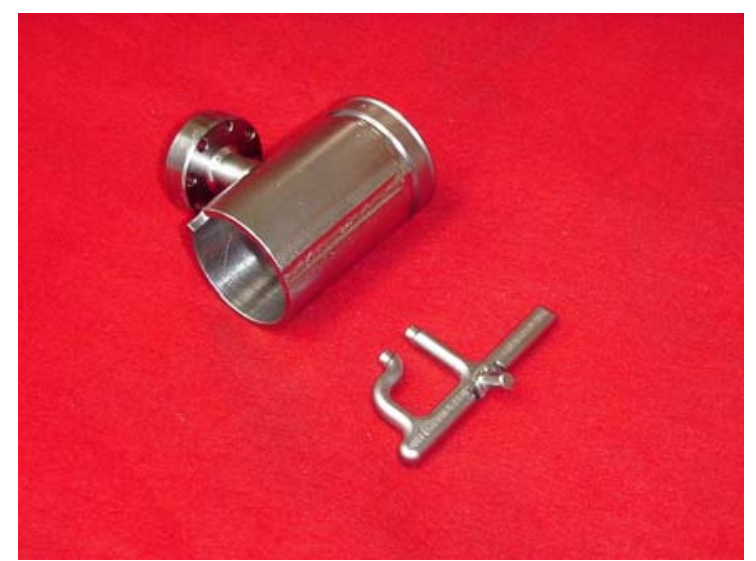

Figure 6: Modified HOM coupler loop

\section{HOM COUPLER TEST CAVITY}

For the evaluation of the modified loop design and various rf feedthroughs, we fabricated a single cell niobium cavity from RRR 250 niobium of the old CEBAF shape with two HOM couplers attached to the beam pipes as shown in Fig. 7. One of the HOM coupler had the old loop configuration, the second coupler was modified as shown in Fig. 6.

Until now we have tested the "in house" feedthrough with a niobium probe tip on the old coupler and a crimped niobium probe tip on a Kyocera feedthrough on the old coupler. Tests of a soldered probe tip on a Kyocera feedthrough on the old and the modified coupler are foreseen in the near future. The results are shown in Figure 7. The "in house" feedthrough was working properly up to $E_{\text {acc }}=30 \mathrm{MV} / \mathrm{m}$; the crimped probe tip on the Kyocera feedthrough "switched " to a lower Q - state at $\mathrm{E}_{\mathrm{acc}} \sim 9 \mathrm{MV} / \mathrm{m}$ in the first test; in a second test after 
some rework on the crimping the "switch" occurred at $E_{\text {acc }}=15.4 \mathrm{MV} / \mathrm{m}$; however, in both cases after switching the rf off, the high Q - state immediately returned, indicating that the heat transfer through the ceramic of the feedthrough is rather good and that possibly the crimping of the probe tip

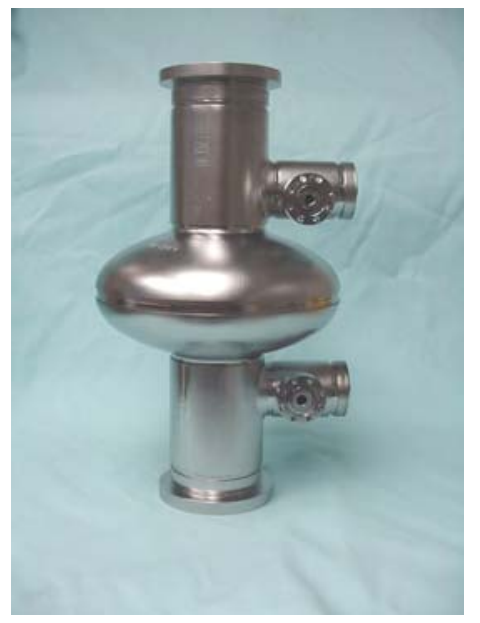

Figure 7: HOM Coupler Test cavity.

was responsible for the field limitation. One has to keep in mind, however, that in a cryomodule the HOM feedthroughs are sitting outside the LHe-bath in vacuum and therefore the cooling conditions are worse. Additional tests under such conditions are needed.

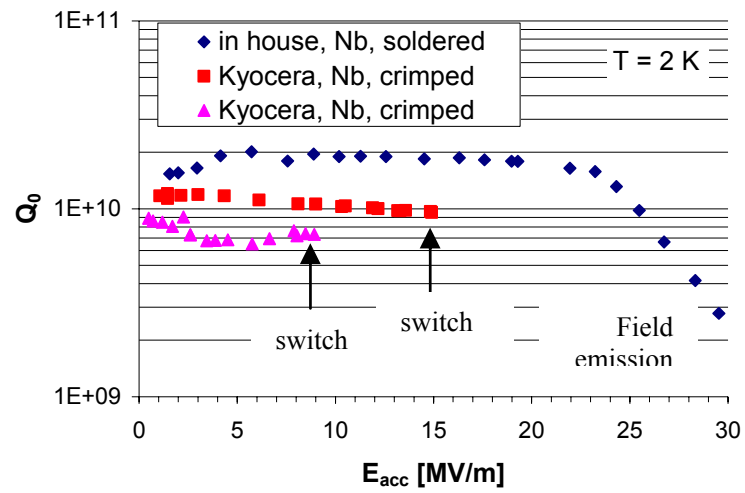

Figure 8: Cavity Performance for different HOMcoupler/feedthrough configurations.

\section{FURTHER IMPROVEMENTS}

If one would replace the niobium probe tip with a material with higher critical temperature $T_{C}$ such as e.g. $\mathrm{Nb}_{3}$ Sn, feedthrough/probe tip configuration would become thermally even more stable. Using measured surface resistances for $\mathrm{Nb}_{3} \mathrm{Sn}$ [5], the temperature at the probe tip has been calculated as a function of dissipated power and is compared to niobium in Figure 9.

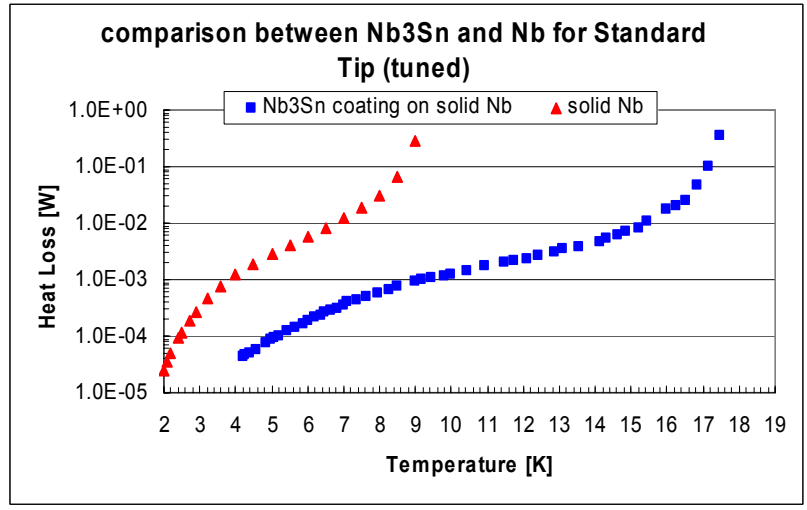

Figure 9: Comparison of calculated heat losses for $\mathrm{Nb}_{3} \mathrm{Sn}$ and $\mathrm{Nb}$ probe tips in standard HOM coupler configuration.

\section{SUMMARY}

A test cavity has been developed, which can be used to evaluate the thermal performance of feedthrough, probe tip and coupler loop configurations in the coaxial HOM coupler. With a feedthrough developed "in house", accelerating gradients up to $E_{\text {acc }}=30 \mathrm{MV} / \mathrm{m}$ could be measured without Q-degradation due to coupler heating. The modified loop as shown in Figure 6 will be tested with a commercially available feedthrough in the near future. Further performance improvement is expected with a higher $\mathrm{T}_{\mathrm{C}}$ material.

\section{ACKNOWLEDGEMENT}

We would like to thank our colleagues B. Manus, G. Slack, L. Turlington, D. Forehand, T. Elliott and R. Bundy from Jlab and G.Kreps from DESY for their support of this work.

\section{REFERENCES}

[1] J. Sekutowicz, Proc. $6^{\text {th }}$ Workshop on RF Superconductivity, Jefferson Lab, Newport News, VA (1993), p.426.

[2] K. Wilson, private communications.

[3] C. Reece, this proceedings.

[4] G. Wu et al., JLAB-TN-04-027.

[5] P. Boccard et al, Proc. $8^{\text {th }}$ Workshop on RF Superconductivity, Abano Terme, Italy (1997), p. 1094. 